\title{
Analysis of optically pumped compact laterally coupled distributed feedback lasers with three symmetric defect regions
}

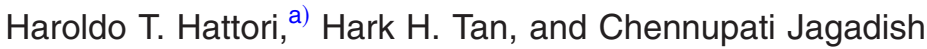 \\ Department of Electronic Materials Engineering, Research School of Physical Sciences and Engineering, \\ The Australian National University, Canberra, Australian Capital Territory 0200, Australia
}

(Received 23 March 2007; accepted 28 August 2007; published online 25 October 2007)

This article analyzes compact laterally coupled distributed feedback (DFB) lasers with three defect regions. These devices are more flexible and smaller than conventional DFB lasers, having typical lengths between 20 and $50 \mu \mathrm{m}$ and a width less than $1 \mu \mathrm{m}$ (lateral gratings are inserted in a single-mode waveguide). We optimize the defect regions to achieve an improved performance. In other words, an adequate choice of phase shifts may lead to single-mode operation, lower threshold optical power, higher quantum differential efficiency, and more uniform field distribution. This device is designed to operate under optical pumping. (C) 2007 American Institute of Physics.

[DOI: $10.1063 / 1.2800285]$

\section{INTRODUCTION}

Distributed feedback (DFB) lasers can provide singlemode operation and narrow spectral linewidths, which might be useful for high speed and large capacity optical networks. These properties are achieved by introducing a wavelength selective corrugation (grating). Many DFB lasers with uniform gratings cannot operate in a single-mode regime, which demands the addition of phase shifts to the uniform structure. A single phase shift of $\pi / 2$ at the center of the corrugation can provide single-mode operation but at the expense of a nonuniform distribution of light in the laser device. This nonuniform distribution of light can lead to spatial hole burning, which can degrade the single-mode operation at higher bias currents. ${ }^{1}$ Moreover, if the electromagnetic fields are distributed over a broader region, the laser device may provide higher output power, better heat dissipation, and narrower beam divergence. ${ }^{2}$ Heat dissipation is a key issue on the performance of compact laser devices and can considerably degrade their performance. A more uniform distribution of light can be achieved by introducing additional phase shifts; three phase shifts seem to provide uniform light distribution in this type of DFB structure. ${ }^{3,4}$ Also, the accumulation of light in a certain region of a laterally coupled DFB (LCDFB) laser might lead to additional radiation losses.

Most of the grating structures employed in conventional DFB lasers are vertical gratings. Recently, DFB lasers with surface lateral gratings have been demonstrated in the literature. ${ }^{5,6}$ They are very flexible in terms of designing the device to operate at a desired wavelength and do not require regrowth, considerably reducing the technological complexity. ${ }^{6}$ Moreover, because of the high index contrast between air and the epilayers, it is possible to design compact laser devices (with lengths less than $50 \mu \mathrm{m}$ and widths less than $1 \mu \mathrm{m}$ ). The high index contrast leads to coupling efficiencies in the order of $100000 \mathrm{~m}^{-1}$.

We have recently analyzed grating structures with symmetric organized gaps. ${ }^{7-9}$ In these structures, the lengths of

\footnotetext{
${ }^{a)}$ Electronic mail: hth109@rsphysse.anu.edu.au
}

the subgratings were integer multiples of a reference phase shift. For example, the three phase shifts introduced in the DFB structure may be $\pi / 3,2 \pi / 3$, and $\pi / 3$ (multiples of $\pi / 3$ ) or $\pi / 4, \pi / 2$, and $\pi / 4$ (multiples of $\pi / 4$ ). With the introduction of these phase shifts, a more uniform electromagnetic field distribution can be obtained. ${ }^{9}$ In this paper, we design and analyze the effect of different phase shifts on the performance of compact LC-DFB lasers, either with three equal or two equal and one distinct phase shifts, following a fractionally ordered rule.

In order to assess the field distribution and resonant wavelengths in these laser structures, we employ a commercial three-dimensional finite-difference time domain (FDTD) software,${ }^{10}$ and to estimate the steady-state laser analysis of a few selected cases, a coupled mode theory is employed. ${ }^{11}$ Based on the simulation results, we have found that an adequate choice of phase shifts can actually lead to better device performance, i.e., a more uniform field distribution, lower threshold power level, lower radiation losses, and single-mode operation. Moreover, these devices are considerably smaller than conventional DFB lasers.

\section{DEVICE DESIGN AND FIELD DISTRIBUTION ANALYSIS}

The structure in which the laser structures will be fabricated is shown in Fig. 1. It consists of an active layer of GaAs with three $7.3 \mathrm{~nm}$ thick $\operatorname{In}_{0.2} \mathrm{Ga}_{0.8}$ As quantum wells, separated by $6 \mathrm{~nm}$ GaAs confinement barriers. The thickness of the active layer $\left(h_{1}\right)$ is about $100 \mathrm{~nm}$. In this active layer, a surface grating with three defect regions is introduced. If the grating structure has a total length $L$, these defect regions are positioned at $z=-L / 4,0$, and $L / 4$. Positioning the defects at other positions generally does not lead to an improved performance. ${ }^{9}$ Below this active layer, an oxidized $\mathrm{Al}_{0.98} \mathrm{Ga}_{0.02} \mathrm{As}$ layer provides good vertical optical confinement for the laser structure. The thickness $\left(h_{2}\right)$ of the oxidized layer is about $450 \mathrm{~nm}$ (before oxidation takes place). These layers are supported by a GaAs substrate. This device shall operate at a free-space wavelength $(\lambda)$ close to $980 \mathrm{~nm}$. 


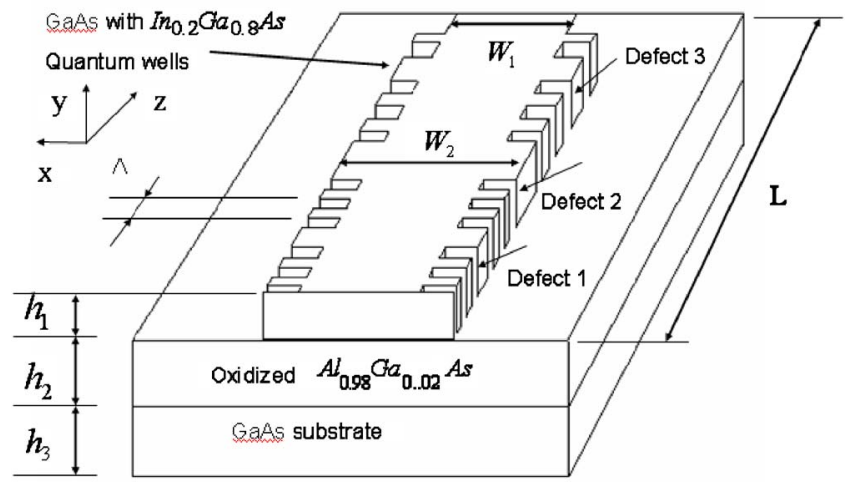

FIG. 1. (Color online) Basic schematic of the laterally coupled distributed feedback structure with three defect regions. Typical values of $L$ range from 20 to $50 \mu \mathrm{m}$.

This structure is primarily designed for laser devices operating under optical pumping; electrically pumped sources would require a different slab structure. This device will be pumped with a pump laser and an optical setup (with lenses, mirrors, detectors, monochromators, etc.) will collect the emitted light, similar to how microdisk and other lasers are conventionally characterized. ${ }^{12}$

The widths of the laser device are $W_{1}=300 \mathrm{~nm}$ and $W_{2}$ $=700 \mathrm{~nm}$, i.e., the surface gratings are $200 \mathrm{~nm}$ wide. The parameter $W_{1}$ is chosen in such a way that only a single transverse mode can propagate inside the DFB laser structure. The surface grating is fabricated by etching away the active region. The surface grating structure has a period $\Lambda$ and $50 \%$ filling factor (area occupied by air divided by the area occupied by the elementary cell). The defect regions are extended "solid" regions and introduce additional phase shifts $\theta_{1}, \theta_{2}$, and $\theta_{3}$.

In order to assess the performance of these devices, a commercial three-dimensional FDTD software ${ }^{10}$ is employed. A source matched to a $300 \mathrm{~nm}$ waveguide is located inside the laser structure and power/field monitors are placed at the ends of the laser structure, at the lateral sides, and on the top and at the bottom of the computation region. ${ }^{13,14}$ The computation region is terminated by absorbing layers. The main propagating mode is assumed to be TE mode (main magnetic field in the $y$ direction, using a standard photonic crystal convention ${ }^{15,16}$ ). In order to operate near $980 \mathrm{~nm}$, the period $\Lambda$ is chosen as $220 \mathrm{~nm}$. In this paper, the phase shifts introduced at the defect regions will be denoted by $\theta_{i}$ $=2 \pi n_{\mathrm{eff}} \Delta L_{i} / \lambda_{\mathrm{Bragg}}$, where $i=1,2,3$ (there are three defects), $n_{\text {eff }}=2.375$ is the effective index of the $300 \mathrm{~nm}$ waveguide, $\Delta L_{i}$ is the "extended" length of the $i$ th $(i=1,2,3)$ defect region, and $\lambda_{\text {Bragg }}=2 n_{\text {eff }} \Lambda$ is the Bragg wavelength of the grating structure.

The first case to be considered here is a DFB structure with no phase shifts, i.e., $\theta_{1}=0, \theta_{2}=0$, and $\theta_{3}=0$. In other words, this structure does not have any defect regions, that is, the structure has only lateral uniform gratings along its extension. This structure will be used as a reference for the analysis of other DFB structures. Initially, the total length of the DFB structure is chosen as $L=20 \mu \mathrm{m}$. The $y$ component of the magnetic field $\left(H_{y}\right)$ spectrum is shown in Fig. 2(a) for a field monitor located at $z=-L / 2$ (back of the DFB laser
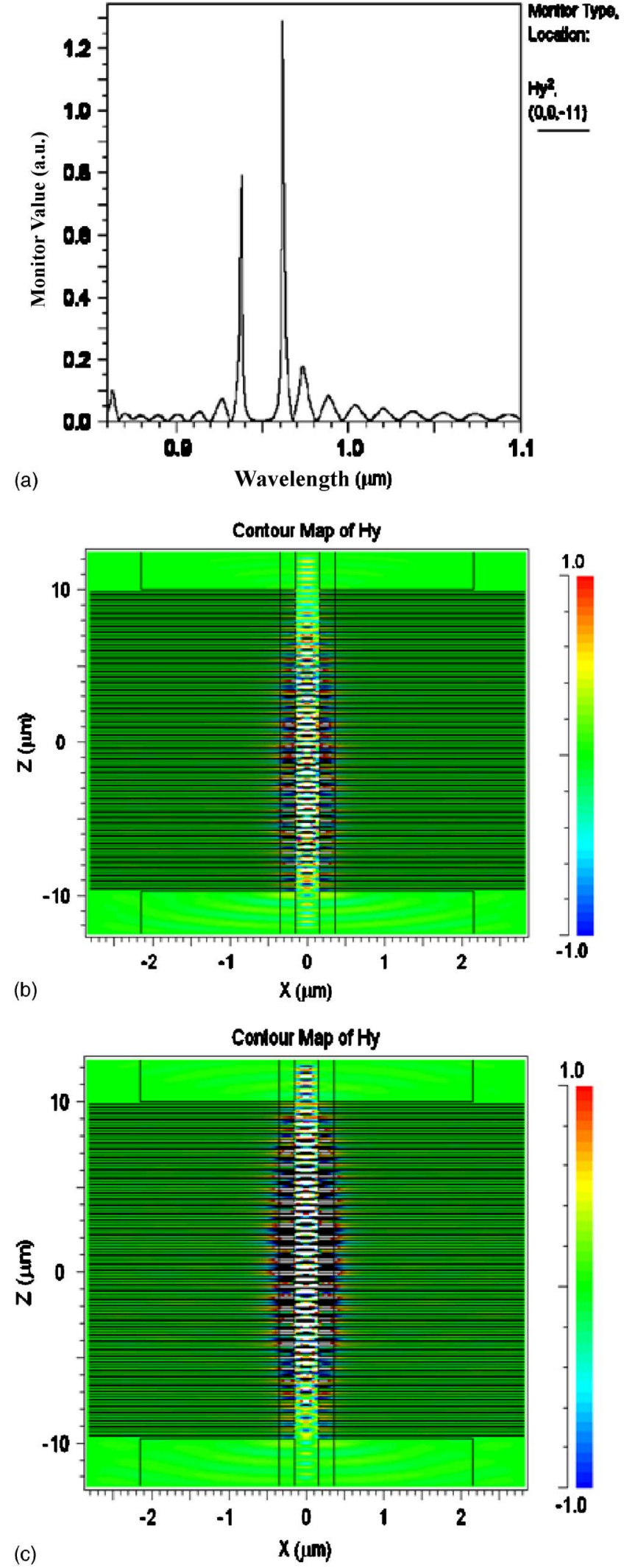

FIG. 2. (Color online) (a) Electromagnetic field spectrum $\left(H_{y}\right)$ at the back of the DFB laser with a uniform grating. (b) Field distribution $\left(H_{y}\right)$ at $\lambda$ $=969.7 \mathrm{~nm}$. (c) Field distribution $\left(H_{y}\right)$ at $\lambda=1020 \mathrm{~nm}$.

structure). There are two main peaks at $\lambda=937.6 \mathrm{~nm}$ and $\lambda$ $=961.8 \mathrm{~nm}$, with quality factors of 1100 and 740, respectively. These two peaks appear close to the edge of the grating band gap, which explains the multitude of other lower 
quality factor $(Q)$ lateral sidebands. Looking at the field distribution of these two main resonant peaks, it can be noticed that there is no huge concentration of electromagnetic field along the $z$ direction. However, this laser device might easily resonate at two distinct wavelengths.

In the second case, we introduce three equal quarterwavelength phase shifts $\theta_{1}=\theta_{2}=\theta_{3}=\pi / 2$. A quarterwavelength phase shift is commonly employed to create optical cavities not only in DFB lasers but also in other laser structures such as distributed Bragg reflector lasers. The defect regions are located at $z=-L / 4, z=0$, and $z=L / 4$. The $y$-component $\left(H_{y}\right)$ magnetic field spectrum at the back of the DFB laser device $(z=-L / 2)$ shows two resonant peaks, one appearing at $\lambda=944.6 \mathrm{~nm}$ and another at $\lambda=956.1 \mathrm{~nm}$, with quality factors of 610 and 500, respectively [Fig. 3(a)]. Both modes have similar quality factors, meaning that they may compete to lase or may lase simultaneously. The field distribution $\left(H_{y}\right)$ at $\lambda=944.6 \mathrm{~nm}$ [Fig. 3(b)] shows that it is no longer uniform and there is accumulation of light near these defect regions. The accumulation of light near the defect regions is more evident in Figs. 3(c) and 3(d), when $\lambda$ $=956.1 \mathrm{~nm}$. In Fig. 3(d), we have increased the number of field levels $(\lambda=956.1 \mathrm{~nm})$ with respect to Fig. 3(c), showing the field distribution with more details. Looking at Fig. 3(d), it looks more evident that accumulation of light in a certain region may lead to additional lateral radiation losses, explaining the lower quality factor at $\lambda=956.1 \mathrm{~nm}$. A power budget analysis shows that about $40 \%$ of the emitted power is lost laterally, $12 \%$ is lost in the vertical direction (5\% in the air region and $7 \%$ in the oxide region), while the remaining power is emitted at the front and back of the $300 \mathrm{~nm}$ waveguide.

One possible way to reduce light accumulation at the defects is to change the phase shifts to $\theta_{1}=\theta_{2}=\theta_{3}=2 \pi / 3$. This has been used to reduce hole burning effects and provide a more uniform field distribution in larger DFB lasers with defect regions. ${ }^{3,9}$ In this case, the $y$ component of the magnetic field $\left(H_{y}\right)$ spectrum [Fig. 4(a)] at the back of the DFB laser device $(z=-L / 2)$ shows two resonant peaks, a main resonant peak at $\lambda=951.2 \mathrm{~nm}$ and a secondary resonant peak at $\lambda=962.2 \mathrm{~nm}$ with quality factors of 600 and 300 , respectively. The field distribution at $\lambda=951.2 \mathrm{~nm}$ is shown in Fig. 4(b). Apparently, this configuration really leads to a more uniform field distribution, but not with a considerably higher quality factor. A power budget analysis indicates that about $30 \%$ of the emitted power is lost laterally, $12 \%$ is emitted in the vertical direction, and the remaining $58 \%$ of the emitted power is emitted at the end regions (back and front) of the DFB laser structure. The field distribution at $\lambda$ $=962.2 \mathrm{~nm}$ is shown in Fig. 4(c). This mode has a low $Q$ and the field distribution shows that apparently the electromagnetic fields are not well confined in the grating region but at the ends of the grating structure, indicating that this may not be a truly resonant mode for the DFB laser structure but a sort of Fabry-Pérot resonance caused by the discontinuity at the interfaces between the grating structure and the waveguides.

So far, the defects in our DFB structures have introduced equal phase shifts. In a recent paper, ${ }^{9}$ we have theoretically
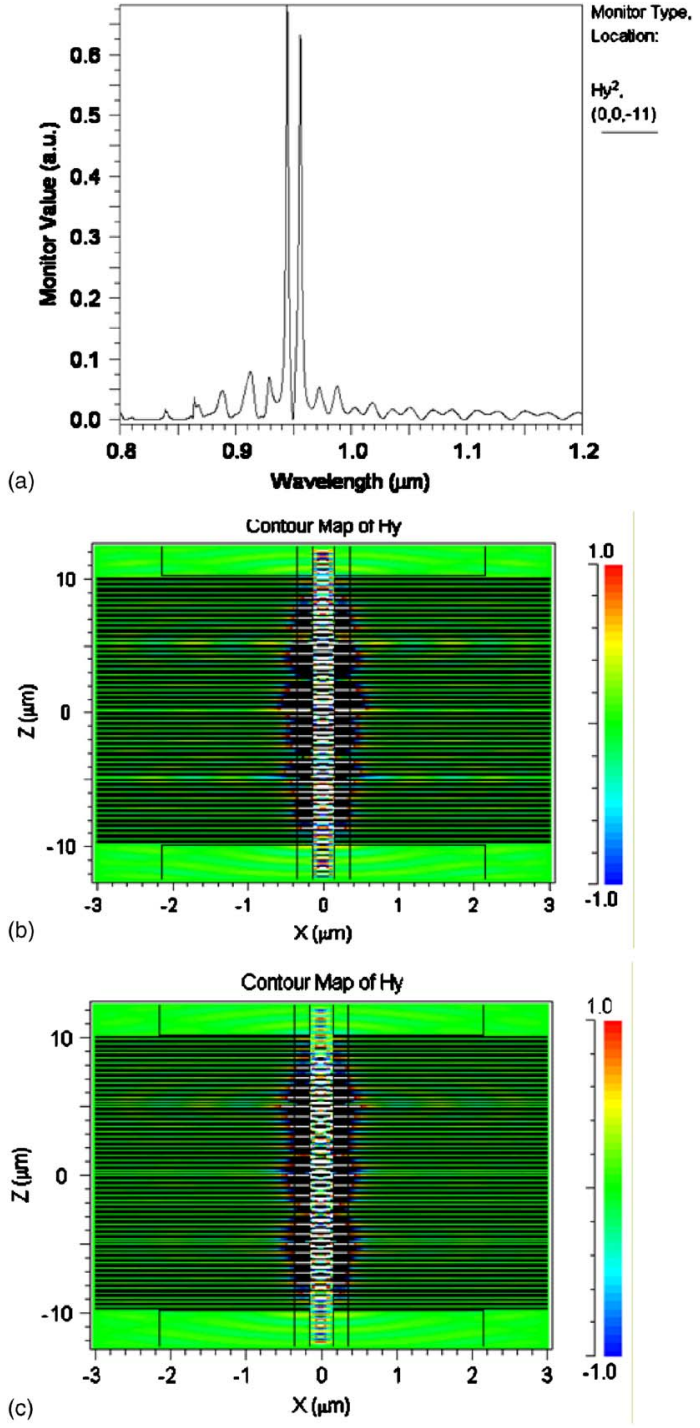

(c)

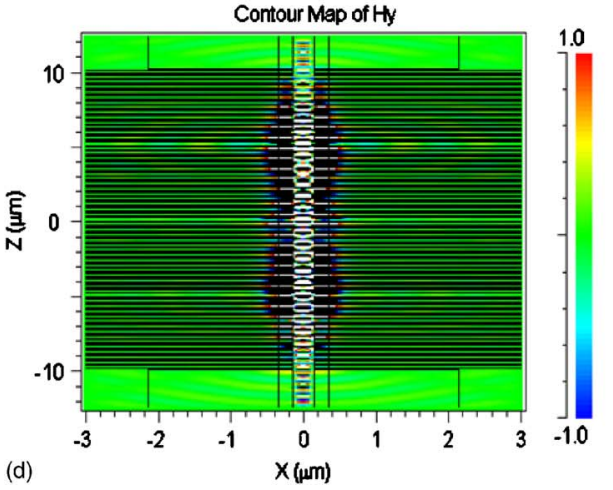

FIG. 3. (Color online) (a) Electromagnetic field spectrum $\left(H_{y}\right)$ at the back of the DFB laser with three phase shifts of $\theta_{1}=\theta_{2}=\theta_{3}=\pi / 2$, evenly distributed along the structure. (b) Field distribution $\left(H_{y}\right)$ at $\lambda=944.6 \mathrm{~nm}$. (c) Field distribution $\left(H_{y}\right)$ at $\lambda=944.6 \mathrm{~nm}$. (d) A more detailed field distribution $\left(H_{y}\right)$ at $\lambda=956.1 \mathrm{~nm}$.

analyzed vertical grating structures with fractionally organized gaps in bulk semiconductors. In that paper, these gratings were able to produce a more uniform field distribution and lower threshold levels. Inspired by those results, we analyze here the performance of laterally coupled DFB lasers with fractionally organized gratings. The first structure to be considered here is one in which $\theta_{1}=\theta_{3}=\pi / 3$ and $\theta_{2}=2 \pi / 3$ 

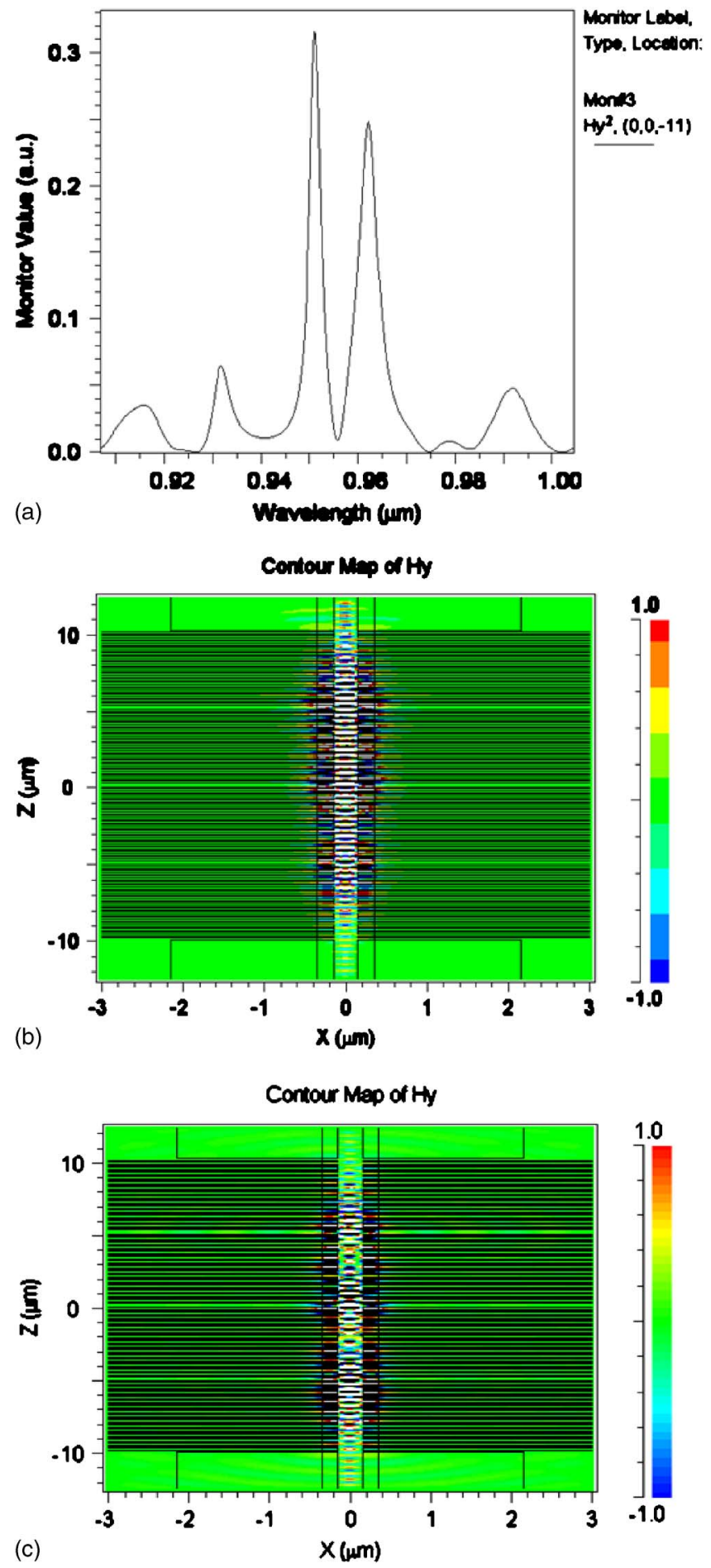

FIG. 4. (Color online) (a) $H_{y}$ spectrum at the back of the DFB laser with three phase shifts of $\theta_{1}=\theta_{2}=\theta_{3}=2 \pi / 3$, evenly distributed along the structure. (b) Field distribution $\left(H_{y}\right)$ at $\lambda=951.2 \mathrm{~nm}$. (c) Field distribution $\left(H_{y}\right)$ at $\lambda=956.1 \mathrm{~nm}$.

(it is a variant of the previous structure, but with $\theta_{1}=\theta_{3}$ $=\theta_{2} / 2=\pi / 3$ ). The $H_{y}$ spectrum at the back end of the grating structure is shown in Fig. 5(a). The main peak appears at $\lambda$ $=957.9 \mathrm{~nm}$, with a quality factor of 700 and apparently this structure is essentially single mode, i.e., there is one main peak due to the grating structure and a few Fabry-Pérot peaks due to the discontinuities at the end of the grating structure (transition waveguide grating). The field distribution at this
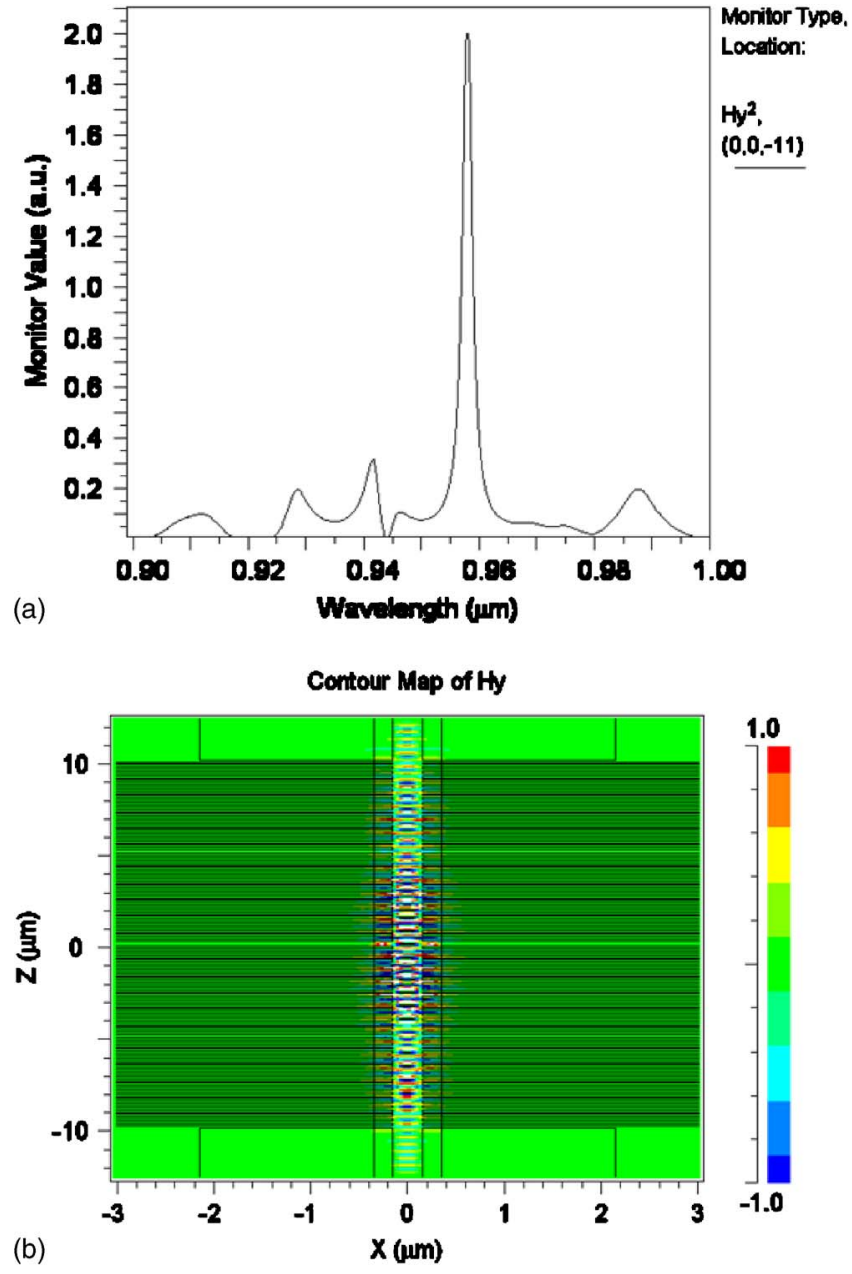

FIG. 5. (Color online) (a) $H_{y}$ spectrum at the back of the DFB laser with three phase shifts of $\theta_{1}=\theta_{3}=\pi / 3$ and $\theta_{3}=2 \pi / 3$. (b) Field distribution $\left(H_{y}\right)$ at $\lambda=957.9 \mathrm{~nm}$.

resonant peak is shown in Fig. 5(b). The field distribution is more uniform than in the case where $\theta_{1}=\theta_{2}=\theta_{3}=\pi / 2$, but with some power concentration at the mid-defect section $\left(\theta_{2}=2 \pi / 3\right)$. A power budget analysis indicates that $12 \%$ of the emitted power is lost in the vertical direction, while $28 \%$ of the power is lost laterally. About $60 \%$ of the emitted power is emitted either in the front or in the back ends of the grating structure, being directly coupled into the $300 \mathrm{~nm}$ ridge waveguides.

A better solution is provided by $\theta_{1}=\theta_{3}=\pi / 4$ and $\theta_{2}$ $=\pi / 2$. The $H_{y}$ spectrum is shown in Fig. 6(a). The main peak appears at $\lambda=952.1 \mathrm{~nm}$ with a quality factor of 1500 . The quality factor for this structure is significantly higher than in the previous cases. Lateral peaks have much smaller quality factor (lower than 400), which might be Fabry-Pérot oscillating peaks at the ends of the DFB structure (the transitions between the grating and the waveguide are not perfectly matched). The field distribution $\left(H_{y}\right)$ in Fig. 6(b) ( $x-z$ plane) shows that there is a concentration of light close to the central grating, but not as strong as when $\theta_{1}=\theta_{2}=\theta_{3}=\pi / 2$, and it looks to be spread over a larger extent ( $z$ direction). There is no major concentration of light close to the regions where the phase shifts are $\theta_{1}=\theta_{3}=\pi / 4$. Apparently, the two defects at $z=-L / 4$ and $z=L / 4$ seem to "attract" light and avoid 

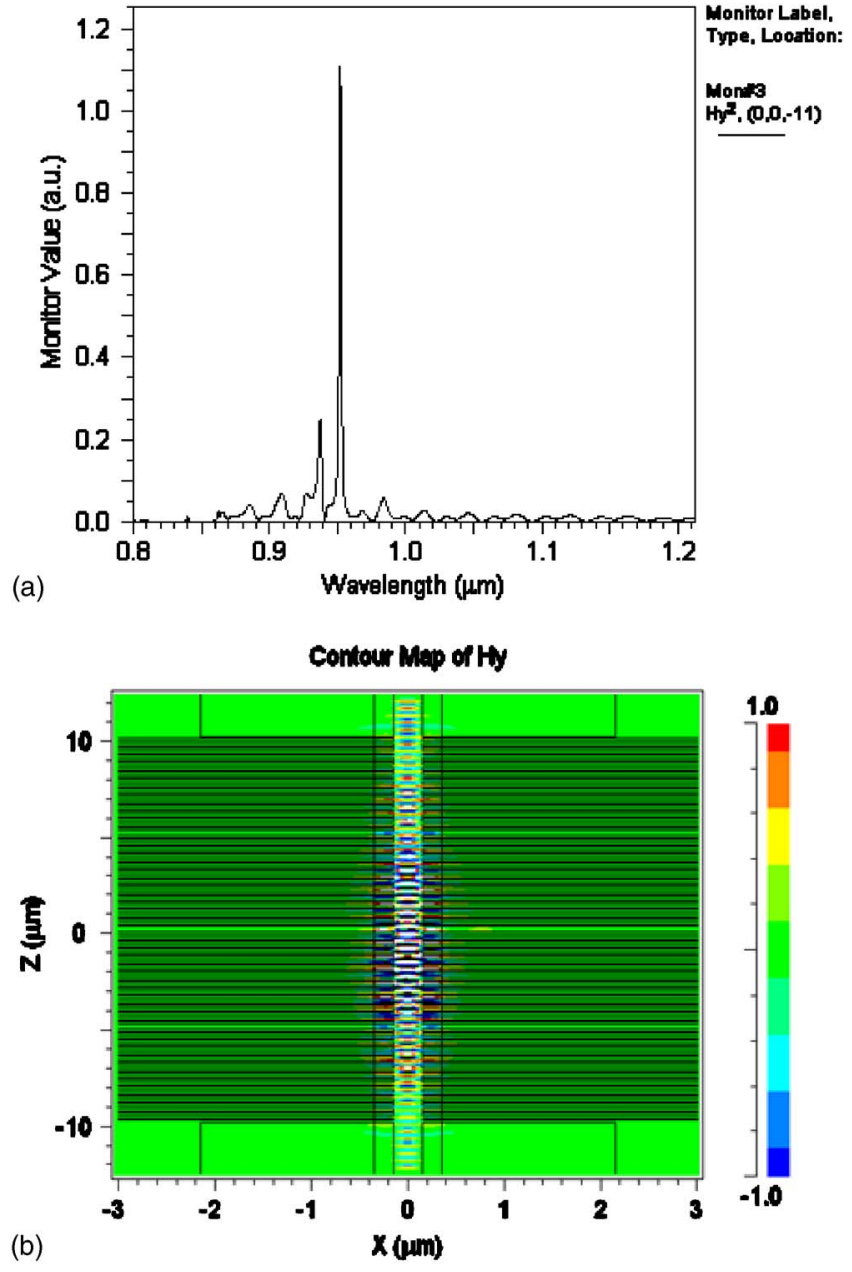

(b)

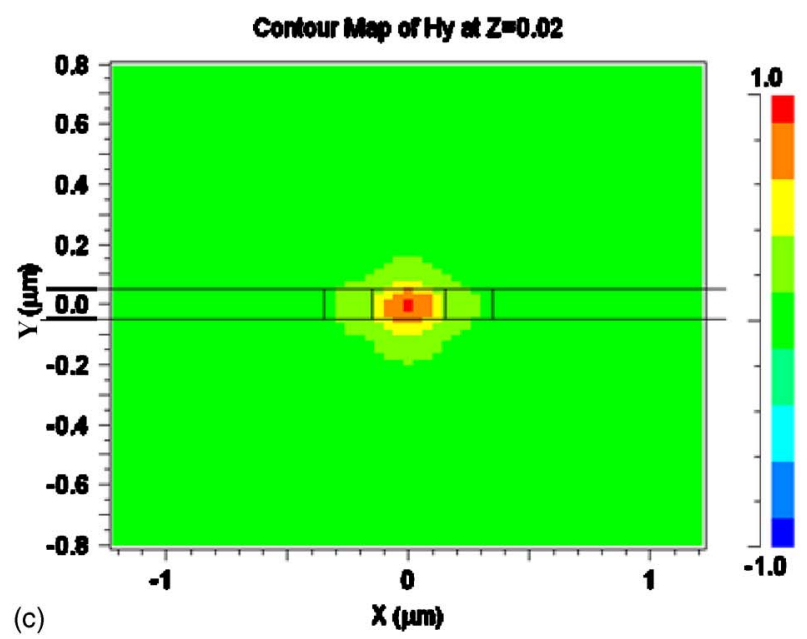

FIG. 6. (Color online) (a) $H_{y}$ spectrum at the back of the DFB laser with three phase shifts of $\theta_{1}=\theta_{3}=\pi / 4$ and $\theta_{3}=\pi / 2$. (b) Field distribution $\left(H_{y}\right)$ at $\lambda=952.1 \mathrm{~nm}(x-z$ plane $)$. (c) Field distribution $\left(H_{y}\right)$ at $\lambda=952.1 \mathrm{~nm}(x-y$ plane).

much light concentration at the middle defect. Light seems to be more localized than in the previous case, but power budget analysis indicates that $12 \%$ of light is emitted in the vertical direction, $12 \%$ of light is lost laterally, while $76 \%$ of light is emitted through the ends of the $300 \mathrm{~nm}$ waveguides. Hence, this configuration provides the best quality factor and lower losses among all three defect regions of LC-DFB structures analyzed here. The cross section $H_{y}$ field distribu- tion is shown in Fig. 6(c) and shows that light is well confined inside the core of the epilayered structure.

If $L$ is increased to $50 \mu \mathrm{m}$, the quality factor for the structure in which $\theta_{1}=\theta_{3}=\pi / 3$ and $\theta_{2}=2 \pi / 3$ increases to 1800 , while the $Q$ factor for the case in which $\theta_{1}=\theta_{3}=\pi / 4$ and $\theta_{2}=\pi / 2$ increases to 3200 . Because of limitations of our current fabrication facilities, it is difficult to fabricate a device longer than $50 \mu \mathrm{m}$; hence we will restrict our analysis to $L<50 \mu \mathrm{m}$.

\section{STEADY-STATE ANALYSIS}

In order to estimate the steady-state characteristics of the laterally coupled laser device, we employ a coupled mode analysis. Although the refractive index contrast in the grating region is high, we believe that the coupled mode theory can still provide a good approximation for the lasing characteristics of these devices. To analyze these laser structures, we divide each of them in more than 10000 spatial steps and use a transfer matrix method (TMM) that can be expressed as $^{11}$

$$
\left[\begin{array}{l}
E_{i}\left(z_{n+1}\right) \\
E_{r}\left(z_{n+1}\right)
\end{array}\right]=\left[\begin{array}{ll}
r_{11}^{n} & r_{12}^{n} \\
r_{21}^{n} & r_{22}^{n}
\end{array}\right]\left[\begin{array}{l}
E_{i}\left(z_{n}\right) \\
E_{r}\left(z_{n}\right)
\end{array}\right],
$$

where $E_{i}\left(z_{n}\right)$ is the electric field propagating in the $+z$ direction at the $n$th section and $E_{r}\left(z_{n}\right)$ is the electric field of the $n$th section propagating in the $-z$ direction. The $n$th section has a length of $z_{n+1}-z_{n}$. The total electric field at the $n$th section is given by $E\left(z_{n}\right)=c_{o}\left[E_{i}\left(z_{n}\right)+E_{r}\left(z_{n}\right)\right]$, where $c_{o}$ is a dimensionless constant. This matrix formulation relates the fields (incident and reflected) at the end of the $n$th section as a function of the fields at the beginning (incident and reflected) of this section and includes the effects of propagation through this section (phase and amplitude changes due to propagation delays and material gain). This matrix takes into account the effects of the corrugation in the presence of the material gain and the transfer matrix method, in general, can include effects of phase and other types of discontinuities, analyze nonuniform gratings, etc. The matrix elements are given by ${ }^{9,11}$

$$
\begin{aligned}
& r_{11}^{n}=\frac{\left(G_{n}-\rho_{n}^{2} G_{n}^{-1}\right) \exp \left[-j \beta_{o}\left(z_{n+1}-z_{n}\right)\right]}{1-\rho_{n}^{2}}, \\
& r_{12}^{n}=\frac{-\rho_{n}\left(G_{n}-G_{n}^{-1}\right) \exp \left(-j \Omega_{n}\right) \exp \left[-j \beta_{o}\left(z_{n+1}+z_{n}\right)\right]}{1-\rho_{n}^{2}}, \\
& r_{21}^{n}=\frac{\rho_{n}\left(G_{n}-G_{n}^{-1}\right) \exp \left(j \Omega_{n}\right) \exp \left[j \beta_{o}\left(z_{n+1}+z_{n}\right)\right]}{1-\rho_{n}^{2}} \\
& r_{22}^{n}=\frac{-\left(\rho_{n}^{2} G_{n}-G_{n}^{-1}\right) \exp \left[j \beta_{o}\left(z_{n+1}-z_{n}\right)\right]}{1-\rho_{n}^{2}}
\end{aligned}
$$

where $\Omega_{n}=0$ is the residue corrugation phase.

$$
G_{n}=\exp \left[\gamma_{n}\left(z_{n+1}-z_{n}\right)\right]
$$




$$
\rho_{n}=\frac{j \kappa_{c}}{\alpha_{n}-j \delta_{n}+\kappa_{c}},
$$

and $\beta_{o}=2 \pi n_{\text {eff }} / \lambda$ is the phase constant. The propagation constant $\gamma_{n}$ is expressed as

$$
\gamma_{n}^{2}=\left(\alpha_{n}-j \delta_{n}\right)^{2}+\kappa_{c}^{2},
$$

where the gain $\left(\alpha_{n}\right)$ and phase $\left(\delta_{n}\right)$ terms of the propagation constant are expressed as

$$
\begin{aligned}
& \alpha_{n}=\frac{\Gamma g-\alpha_{\text {loss }}}{2}, \\
& \delta_{n}=\frac{2 \pi n_{\text {eff }}}{\lambda}-\frac{2 \pi n_{\mathrm{gr}}\left(\lambda-\lambda_{\text {Bragg }}\right)}{\lambda \lambda_{\text {Bragg }}}-\frac{\pi}{\Lambda} .
\end{aligned}
$$

It is assumed that the subgratings between the defect regions have similar properties under no bias. Our devices will employ three $7.3 \mathrm{~nm}$ thick $\mathrm{In}_{0.2} \mathrm{Ga}_{0.8}$ As quantum wells, separated by two $6 \mathrm{~nm}$ barrier layers. The optical confinement factor $\Gamma$ can be estimated as $\Gamma=0.22$, the power loss coefficient as $\alpha_{\text {loss }}=4000 \mathrm{~m}^{-1}$, the effective index as $n_{\text {eff }}$ $=2.38$, the group refractive index as $n_{\mathrm{gr}}=3.50$, and the Bragg wavelength as $\lambda_{\text {Bragg }}=950 \mathrm{~nm}$. The quantum well gain can be approximated as ${ }^{17}$

$$
g=g_{o} \ln \left(\frac{N}{N_{\text {tr }}}\right)
$$

where $g_{o}=1.2 \times 10^{5} \mathrm{~m}^{-1}$ is the peak gain and $N_{\mathrm{tr}}=1.8$ $\times 10^{24} \mathrm{~m}^{-3}$ is the transparency carrier concentration. The coupling coefficient $\kappa_{c}$ is calculated by the following formula: ${ }^{11}$

$$
\kappa_{c}=\frac{\pi}{\lambda n_{\mathrm{eff}}} \frac{\iint_{S} A_{1}(x, y)|\mathbf{E}(x, y, z)|^{2} d x d y}{\iint_{S}|\mathbf{E}(x, y, z)|^{2} d x d y},
$$

where $\mathbf{E}(x, y, z)$ is the total electric field distribution (under no bias) in the $300 \mathrm{~nm}$ waveguide in the absence of the grating structure. It is assumed that the grating structure will introduce an electric permittivity perturbation to the $300 \mathrm{~nm}$ waveguide that can be expressed as

$$
\Delta \varepsilon=\sum_{-\infty}^{\infty} A_{q}(x, y) \exp \left(\frac{2 j q \pi z}{\Lambda}\right) .
$$

When an additional phase shift is introduced at the border of the $m$ th section, the transfer matrices (after and before the phase shift) are linked up at the phase shift position as

$$
\left[\begin{array}{c}
E_{i}\left(z_{m}^{+}\right) \\
E_{r}\left(z_{m}^{+}\right)
\end{array}\right]=\left[\begin{array}{cc}
\exp \left(j \theta_{m}\right) & 0 \\
0 & \exp \left(-j \theta_{m}\right)
\end{array}\right]\left[\begin{array}{l}
E_{i}\left(z_{m}^{-}\right) \\
E_{r}\left(z_{m}^{-}\right)
\end{array}\right],
$$

where $z_{m}^{-}$and $z_{m}^{+}$are the positions before and after the phase shift $\theta_{m}$. Assuming that $E_{r}(L / 2)=y_{21} E_{i}(-L / 2)+y_{22} E_{r}(-L / 2)$ and $E_{i}(L / 2)=y_{11} E_{i}(-L / 2)+y_{12} E_{r}(-L / 2)$ (the complete structure starts at $z=-L / 2$ and ends at $z=L / 2)$, the threshold condition is given by ${ }^{11}$

$$
y_{22}+\hat{r}_{1} y_{21}-\hat{r}_{2} y_{12}-\hat{r}_{1} \hat{r}_{2} y_{11}=0,
$$

where $\hat{r}_{1}$ and $\hat{r}_{2}$ are the electric field reflectivities at the ends of the grating structure (front and back of the DFB laser device). This threshold condition allows us to calculate the normalized gain parameter $\left(\alpha_{n} L\right)$ as a function of $\lambda$ for distinct structures. This equation is solved by using NewtonRaphson methods.

A general threshold analysis for several vertical grating DFB structures has already been analyzed by Hattori et al. ${ }^{9}$ so we will restrict our attention to the situation where the gratings are located at $z=-L / 4, z=0, z=L / 4$ and $\theta_{1}=\theta_{3}$ $=\pi / 3$ and $\theta_{2}=2 \pi / 3$ (case 1 , dashed curve) or $\theta_{1}=\theta_{3}=\pi / 4$ and $\theta_{2}=\pi / 2$ (case 2, solid curve). These two structures were selected because they provide high quality factors and can operate under a single longitudinal mode. In order to reduce threshold optical power levels, we will consider $L=50 \mu \mathrm{m}$. A longer laterally coupled DFB structure would be difficult to fabricate with our fabrication facilities. Assuming that this device will be optically pumped, the steps leading to the calculation of the steady-state laser response can be summarized as follows:

(1) For a given wavelength, the threshold normalized gain $\left(\alpha_{n} L\right)$ is determined. From Eqs. (5a) and (6), we can determine the threshold material gain and, consequently, the threshold carrier density $\left(N_{\mathrm{th}}\right)$.

(2) The threshold optical pump power is given by

$$
P_{\mathrm{th}}=\frac{h c V_{a}}{\eta_{p} \lambda_{p}}\left(\frac{N_{\mathrm{th}}}{\tau_{A}}+B N_{\mathrm{th}}^{2}+C N_{\mathrm{th}}^{3}\right),
$$

where $h$ is the Planck's constant, $c$ is the free-space light speed, $V_{a}$ is the volume of the active region $\left(V_{a}=L h_{a} 300\right.$ $\times 10^{-9}, h_{a}$ is the thickness of the active region containing the quantum wells), $\eta_{p}$ is the optical pumping absorption efficiency, $\lambda_{p}=532 \mathrm{~nm}$ is the pump wavelength, $\tau_{A}=4.0 \mathrm{~ns}$ is the linear recombination lifetime, $B=10^{-16} \mathrm{~m}^{3} / \mathrm{s}$ is the radiative spontaneous emission coefficient, and $C=3$ $\times 10^{-41} \mathrm{~m}^{6} / \mathrm{s}$ is the Auger recombination coefficient.

(3) The laser cavity is divided into a large number of sections, each represented by a transfer matrix. It is assumed that the total electric field is given by $E(z)$ $=c_{o}\left[E_{r}(z)+E_{i}(z)\right]$. Initial values of $\lambda$ and $c_{o}$ are chosen to start the numerical procedure. It is assumed that $\left|E_{i}^{2}(z=-L / 2)\right|+\left|E_{r}^{2}(z=-L / 2)\right|=1$.

(4) Initially, the threshold refractive index is used to calculate the transfer matrices for different sections [the refractive effective index is assumed to be $n(z)$ and dependent on $z$, because it depends on the carrier concentration $^{11}$ ]. The carrier concentration $(N)$ is calculated by solving the following equation:

$$
\frac{\eta_{p} P_{p}}{\left(h c / \lambda_{p}\right) V_{a}}=\frac{N}{\tau_{A}}+B N^{2}+C N^{3}+\Gamma \frac{v_{\mathrm{gr}} g_{o} \ln \left(N / N_{\mathrm{tr}}\right) S}{1+\varepsilon S},
$$

where $P_{p}$ is the input pump power, $v_{\mathrm{gr}}=3 \times 10^{8} / n_{\mathrm{gr}} \mathrm{m} / \mathrm{s}$ is the group velocity, $\varepsilon_{p}=1.5 \times 10^{-23} \mathrm{~m}^{3}$ is the gain compression factor, and $S$ is the local photon density. The local photon density is given by

$$
S=\frac{2 \varepsilon_{o} n(z) n_{\mathrm{gr}} \lambda}{h c} c_{o}^{2}\left[\left|E_{i}(z)\right|^{2}+\left|E_{r}(z)\right|^{2}\right] .
$$

Based on this transfer matrix method, it is possible to calculate the electric fields at the right output plane. If they satisfy 
the boundary condition, the procedure is completed, otherwise new values of $\lambda$ and $c_{o}$ are chosen and the interaction is restarted.

(5) The power emitted at the facets are given by

$$
\begin{aligned}
& P_{\text {back }}=h_{1} W_{1} v_{\mathrm{gr}} \frac{h c}{\lambda} S(z=-L / 2), \\
& P_{\text {front }}=h_{1} W_{1} v_{\mathrm{gr}} \frac{h c}{\lambda} S(z=L / 2) .
\end{aligned}
$$

The optical pump absorption efficiency $\left(\eta_{p}\right)$ depends on how the DFB laser structure is being pumped by an external laser. For example, if we consider that the DFB laser device will be pumped by an external laser device with spot size diameter $\phi_{p}, \eta_{p}$ can be approximated by

$$
\eta_{p}=\frac{4 W_{1}}{\pi \phi_{p}} .
$$

Spot size diameters of 2, 5, and $10 \mu \mathrm{m}$ will lead to pump absorption efficiencies of $19 \%, 8 \%$, and $4 \%$, respectively. Hence, output power calculations (at the front end of the DFB laser device, denoted as $P_{\text {front }}$ ) will take into account a few distinct absorption efficiencies. In general, because of the symmetry of the DFB laser structure, we have obtained $P_{\text {front }}$ approximately equal to $P_{\text {back }}$ (power emitted from the back end of the DFB laser device).

The curves showing $P_{\text {front }}$ as a function of the input pump power for distinct values of $\eta_{p}$ are shown in Figs. 7(a) and 7(b) for cases 1 and $2(L=50 \mu \mathrm{m})$. In general, the threshold power levels are more than twice lower in case 2 than in case 1. Also, the differential quantum efficiencies in case 2 are higher than in case 1 . In summary, the laser structure with $\theta_{1}=\theta_{3}=\pi / 4$ and $\theta_{2}=\pi / 2$ seems to provide a better performance when compared with the remaining laser devices considered here and without major concentration of light along the DFB laser structure.

We are currently in the process of fabricating these structures with focused ion beam milling system. However, at this stage, we present our theoretical results concerning these devices.

\section{CONCLUSIONS}

In this paper we have analyzed the field distribution in laterally coupled distributed feedback laser structures with three defect regions. We have considered two basic structures: Structures with three defects that introduce equal phase shifts and symmetrical structures in which the phase shifts at the end of the structure are half of the phase shift introduced by the middle defect. We have observed that concentration of light at a certain defect might lead to additional radiation losses, but with a careful choice of these defects, these losses can be reduced.

A structure with defects located at $z=-L / 4, z=0, z$ $=L / 4$ (where $L$ is the total length of the grating structure) with phase shifts of $\theta_{1}=\theta_{3}=\pi / 4$ and $\theta_{2}=\pi / 2$ has a considerably higher quality factor than other structures. Moreover, light does not seem to be very concentrated at the central
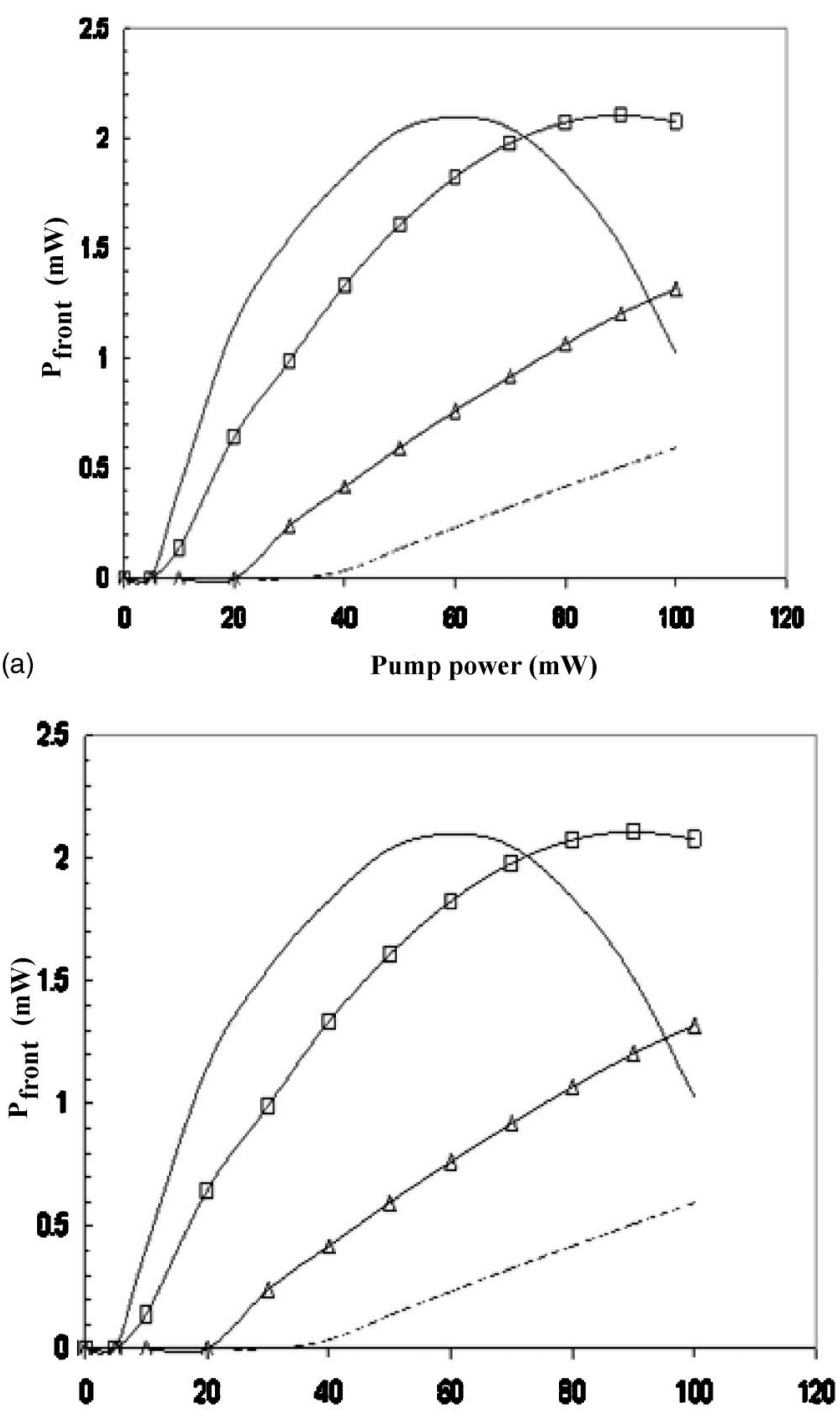

(b)

Pump power $(\mathrm{mW})$

FIG. 7. Emitted power at the front end of the LC-DFB laser device as a function of the input pump power for (a) $\theta_{1}=\theta_{3}=\pi / 3$ and $\theta_{3}=2 \pi / 3$ and (b) $\theta_{1}=\theta_{3}=\pi / 4$ and $\theta_{3}=\pi / 2$. The solid curves with no marks, square marks, and triangular marks are for $\eta_{p}=30 \%, 20 \%$, and $8 \%$, respectively. The dashed curve is for $\eta_{p}=4 \%$.

defect and it might be single-mode longitudinally. This structure has a lower threshold power and a higher differential quantum efficiency.

\section{ACKNOWLEDGMENTS}

The authors wish to gratefully acknowledge the Australian Research Council (ARC) for the financial support of this work.

${ }^{1}$ H. Soda, Y. Kotaki, H. Sudo, H. Ishikawa, S. Yamakoshi, and H. Imai, IEEE J. Quantum Electron. 23, 804 (1987).

${ }^{2}$ D. Ohnishi, T. Okano, M. Imada, and S. Noda, Opt. Express 12, 1562 (2004).

${ }^{3}$ H. Ghafouri-Shiraz and B. Lo, Semicond. Sci. Technol. 8, 1126 (1994).

${ }^{4}$ J. E. A. Whiteaway, B. Garret, G. H. B. Thompson, A. J. Collar, C. J. Armstead, and M. J. Fice, IEEE J. Quantum Electron. 28, 1272 (1992).

${ }^{5}$ H. Abe, S. G. Ayling, J. H. Marsh, R. M. De La Rue, and J. S. Roberts, IEEE Photonics Technol. Lett. 7, 452 (1995).

${ }^{6}$ F. Pozzi, R. M. De La Rue, and M. Sorel, IEEE Photonics Technol. Lett. 18, 2563 (2006) 
${ }^{7}$ C. L. Barbosa, R. M. Cazo, and H. T. Hattori, Microwave Opt. Technol. Lett. 31, 223 (2001).

${ }^{8}$ H. T. Hattori, V. M. Schneider, and O. Lisboa, J. Opt. Soc. Am. A 17, 1583 (2000).

${ }^{9}$ H. T. Hattori, V. M. Schneider, and C. L. Barbosa, Appl. Opt. 46, 1283 (2007).

${ }^{10}$ FULLWAVE 4.0, RSOFT Group, 1999 (http://www.rsoftdesign.com).

${ }^{11}$ H. Ghafouriz-Shiraz, Distributed Feedback Laser Diodes and Optical Tunable Filters (Wiley, New York, 2003).

${ }^{12}$ H. T. Hattori, E. Touraille, C. Seassal, P. Rojo-Romeo, X. Letartre, G. Hollinger, M. Heitzmann, L. Mollard, E. Jalaguier, J. M. Fedeli, and P.
Viktorovitch, IEEE Photonics Technol. Lett. 18, 223 (2006).

${ }^{13}$ H. T. Hattori, C. Seassal, X. Letartre, P. Rojo-Romeo, J. L. Leclercq, P. Viktorovitch, M. Zussy, L. di Cioccio, L. El Melhaoui, and J. M. Fedeli, Opt. Express 13, 3310 (2005).

${ }^{14}$ H. T. Hattori, I. McKerracher, H. H. Tan, C. Jagadish, and R. M. De La Rue, IEEE J. Quantum Electron. 43, 279 (2005).

${ }^{15}$ S. G. Johnson and J. Joannopoulos, Opt. Express 8, 173 (2001).

${ }^{16}$ S. H. Kim, H. Y. Ryu, H. G. Park, G. H. Kim, Y. S. Choi, Y. H. Lee, and J. S. Kim, Appl. Phys. Lett. 81, 2499 (2002).

${ }^{17}$ L. A. Coldren and S. W. Corzine, Diode Lasers and Photonic Integrated Circuits (Wiley, New York, 1995). 